\title{
Patterned Honeycomb Structural Films with Fluorescent and Hydrophobic Properties
}

\author{
Liping Heng, ${ }^{1}$ Rongrong Hu, ${ }^{2}$ Sijie Chen, ${ }^{2}$ Jie Li, ${ }^{2}$ Lei Jiang, ${ }^{1}$ and Ben Zhong Tang ${ }^{2}$ \\ ${ }^{1}$ School of Chemistry and Environment, Beihang University, Beijing 100191, China \\ ${ }^{2}$ Department of Chemistry, State Key Laboratory of Molecular Neuroscience, Institute of Molecular Functional Materials, \\ The Hong Kong University of Science \& Technology, Clear Water Bay, Kowloon, Hong Kong
}

Correspondence should be addressed to Liping Heng; henglp@iccas.ac.cn

Received 19 August 2013; Revised 13 September 2013; Accepted 13 September 2013

Academic Editor: Amir Kajbafvala

Copyright (c) 2013 Liping Heng et al. This is an open access article distributed under the Creative Commons Attribution License, which permits unrestricted use, distribution, and reproduction in any medium, provided the original work is properly cited.

\begin{abstract}
We have fabricated the patterned porous honeycomb-like film with the aggregation-induced emission (AIE) property successfully by breath figure method. Characterization of contact angles (CA) indicated that the patterned porous honeycomb structure can improve the hydrophobicity of the film, while smooth films do not have this function. Characterization of the fluorescence property indicates that the patterned honeycomb structure films are highly emissive. This work could not only be promising for controlling molecular group reorientation and suitable for the application of manipulating surface composition of the film but also make the tetraphenylethene derivatives with AIE properties have a promising application future in special wettability surface. The present findings should open a way for the new application of honeycomb structure materials, which will be useful in many areas, such as sensors, tissue engineering, clinical medicine, and biomaterials.
\end{abstract}

\section{Introduction}

The breath figure $(\mathrm{BF})$ technique is a simple and robust method to prepare porous honeycomb structure films, which was firstly reported by Widawski et al. [1]. The formation mechanism of the honeycomb structure has been suggested by Maruyama et al. [2]. Here, it will be only briefly outlined. When a water-immiscible organic solvent starts to evaporate, its surface becomes cool. Water from the atmosphere starts to condense. Because the condensation takes place on an unstructured liquid surface, the water droplets have a narrow size distribution. With ongoing evaporation of the organic solvent, these water droplets can grow until the solution becomes too viscous. The droplets of the condensed water self-assemble into a hexagonally ordered array at the air/solution interface. After the evaporation of solvent and water droplets, the well-ordered honeycomb structure is left on the film surface. This method is more competitive compared to other methods because it is nonpolluting, cheaper, and faster. In the past ten years, most work in this field has mainly focused on changing polymer pieces and solvents to prepare ordered porous films by the BF process
[3-6]. Recently, research in this field started to concentrate on fabricating new structures, such as patterned structures and three-dimensional structures $[7,8]$, and building new functional honeycomb films with different properties, such as photoelectric conversion [9], photocatalysis [10], antireflection [11], hydrophobicity [12], high mechanical strength $[13,14]$, and cell adhesion [15-17]. However, their new applications, particularly in the aspects of preparing patterned honeycomb structural square lattices, are still in their infancy.

In general, conventional chromophoric molecules are emissive in their dilute solutions but become weakly emissive or even nonemissive in the solid state because aggregation commonly quenches light emission [18]. This phenomenon is called aggregation-caused quenching (ACQ) [19-21], which is very common and has been attributed to the nonradiative decay of sandwich-shaped excimers and exciplexes formed among the closely packed dye molecules in the aggregates. Recently, an extraordinary phenomenon of aggregationinduced emission (AIE) $[22,23]$ was discovered, which is exactly opposite to the ACQ effect discussed above [19-21]. In this paper, we have prepared patterned honeycomb structural square lattices using the polymer with the AIE property and 


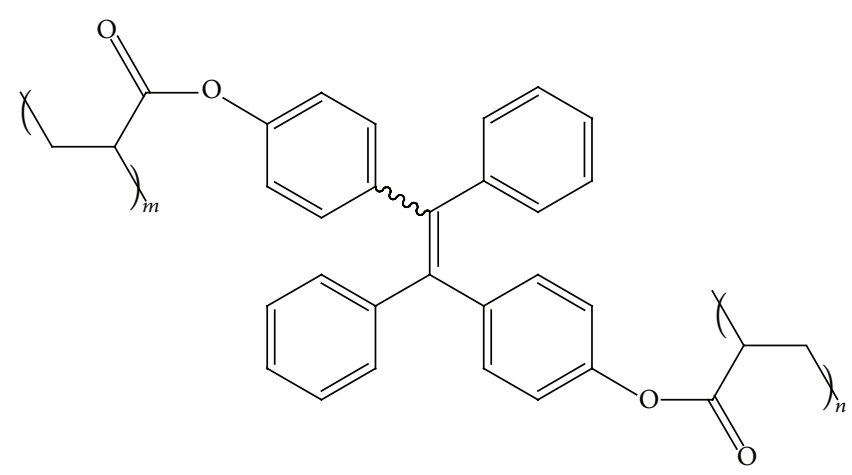

FIGURE 1: Molecular structure of the photo-cross-linking polymer.

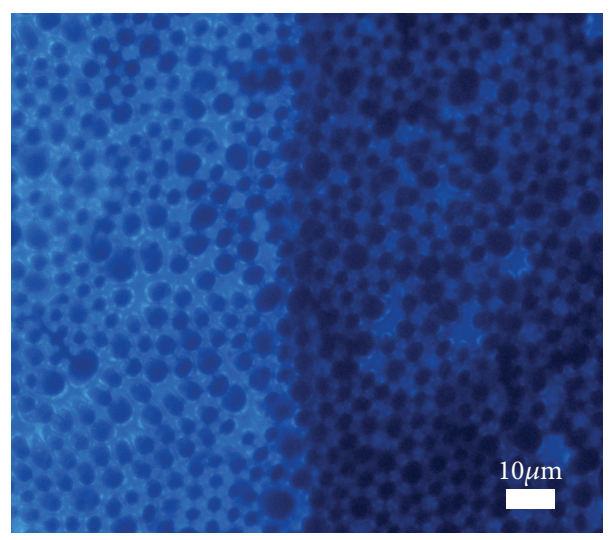

(a)

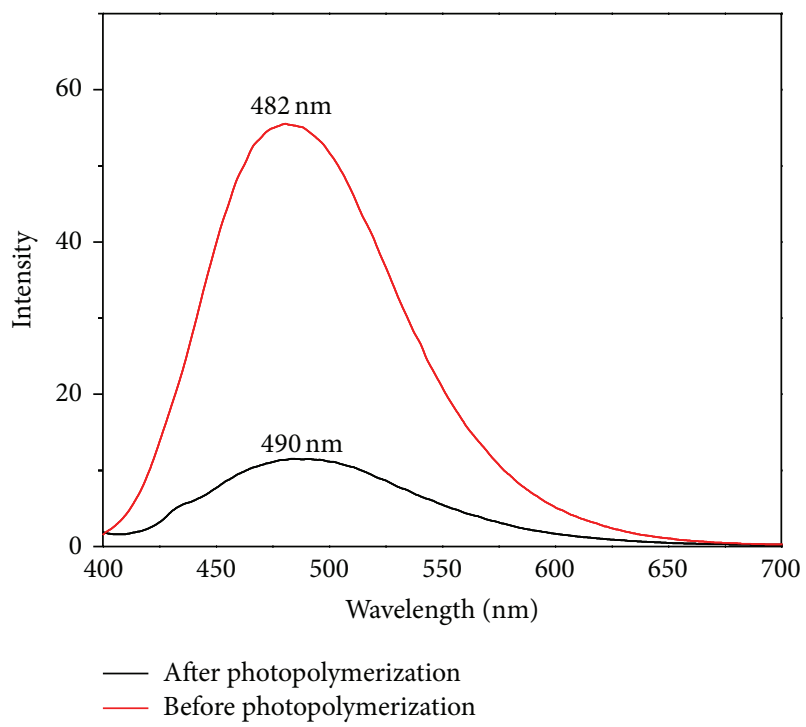

(b)

FiguRE 2: (a) The fluorescence microscope image of the film before photopolymerization (left) and after photopolymerization (right); (b) fluorescent spectrum of the film before and after photopolymerization. Excitation: $320 \mathrm{~nm}$. From the fluorescence microscope image and the fluorescent spectrum of the film before and after photopolymerization, it can be seen that the porous film can be prepared over a large area, which has the AIE property whether it is photopolymerizable or not.

studied their wettability and fluorescence. Characterization of contact angles (CA) on the patterned film indicates that the porous honeycomb structure can improve the hydrophobicity of the film, while smooth films do not have this function. This phenomenon of honeycomb structure will be useful for controlling molecular group reorientation and suitable for the application of manipulating surface composition of the film. Characterization of the fluorescence property indicates that the patterned honeycomb structure film is highly emissive.

\section{Experimental Section}

2.1. Preparation of the Honeycomb Structure. The polymer was provided by HKUST. Its structure was characterized by standard spectroscopic methods. The molecule was characterized by NMR spectroscopy. The purity was also confirmed by elemental analysis with satisfactory results. Water was purified using a Milli-Q purification system (Millipore Corp., Bedford, MA) to give a resistivity of $18 \mathrm{M} \Omega \mathrm{cm}$.

A solution of polymer in 1,2-dichloroethane $(10 \mu \mathrm{L}$, $0.62 \mathrm{wt} \%$ ) was cast on cleaned substrates (glass) at room temperature $\left(20 \pm 1^{\circ} \mathrm{C}\right)$ under a humid atmosphere with relative humidity (R.H.) of $85 \%$, and honeycomb-patterned films were obtained. For comparison, smooth films were fabricated by casting the same solution on substrates under ambient atmosphere (R.H. $=48 \%$ at $20 \pm 1^{\circ} \mathrm{C}$ ).

2.2. Photopatterning. Photo-cross-linking reactions of the polymer films were conducted in air at room temperature using $365 \mathrm{~nm}$ light obtained from a Spectroline ENF-280C/F UV lamp at a distance of $5 \mathrm{~cm}$ from the light source. The incident light intensity was $18.5 \mathrm{~mW} \mathrm{~cm}^{2}$. The photoresist patterns were generated by UV irradiation of the polymer films through copper photomasks for 20 min followed by development in 1,2-dichloroethane. 


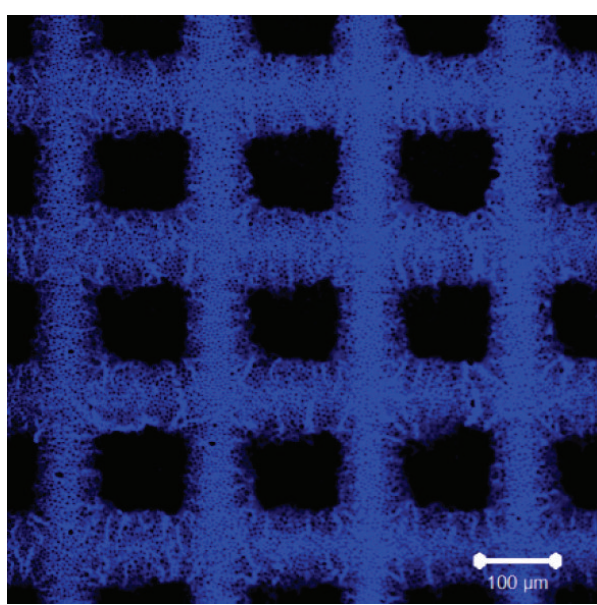

(a)

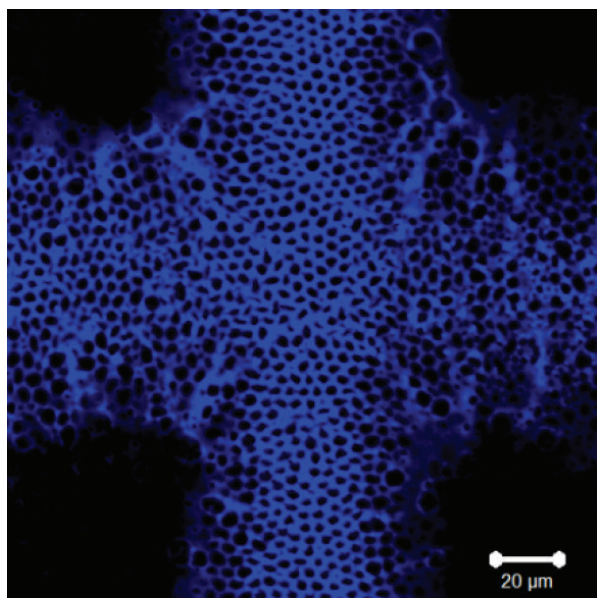

(c)

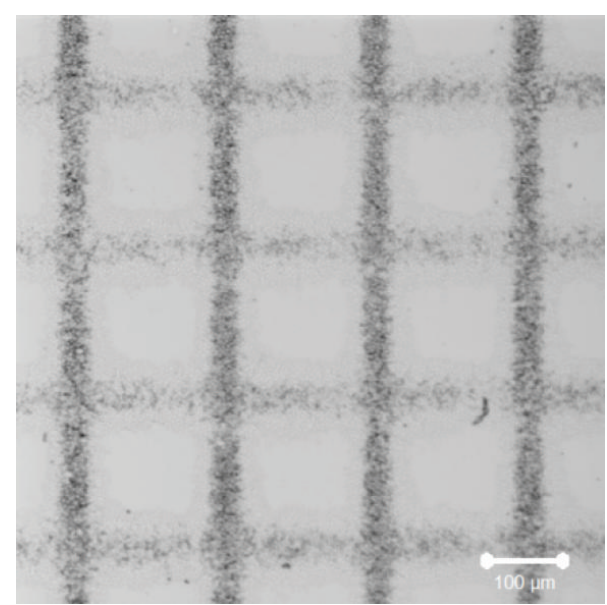

(b)

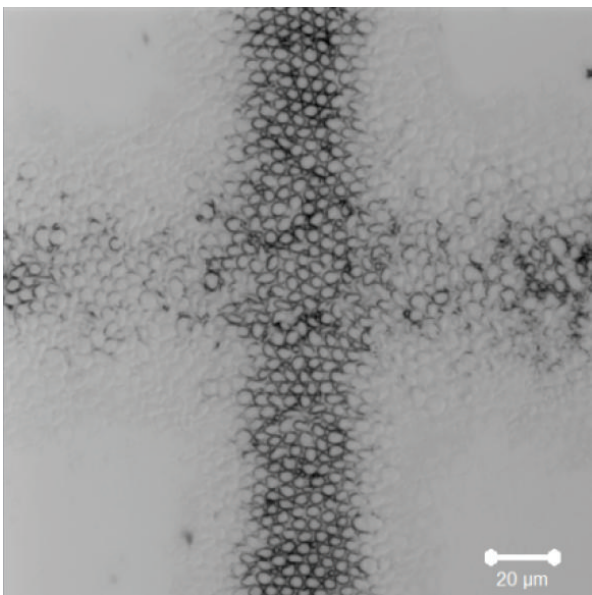

(d)

FIGURE 3: Confocal images of the patterned honeycomb square lattices: (a) low magnification, (c) high magnification, and the corresponding optical photos: (b) low magnification, (d) high magnification. The size of the used mask is $50 \mu \mathrm{m}$ and the space is $150 \mu \mathrm{m}$.

2.3. Sample Characterization. Fluorescence images of the honeycomb film were measured in the Zeiss laser scanning confocal microscope with $405 \mathrm{~nm}$ excitation. Model is LSM7 DUO (710 + LIVE). The contact angles (CA) were examined on a Dataphysics OCA20 CA system at ambient temperature. The CA values were the averages of five independent measurements. The honeycomb structure films were imaged under an inverted fluorescence microscope (Nikon Eclipse TE2000-U), diachronic mirror $400 \mathrm{~nm}$. The images were captured using a digital CCD camera. Fluorescence spectra were tested on a Hitachi F-4500 fluorescence spectrophotometer.

\section{Results and Discussion}

The molecular structure of the polymer used in experiments is shown in Figure 1, and the molecular weight is 19600 [24]. The polymer molecule contains tetraphenylethene (TPE) structural unit, which is a kind of typical nonplanar molecule with the AIE property. When it is integrated to the conventional dye molecules, it would twist the adduct molecules with significantly decreased effect of intramolecular $\pi-\pi$ stacking in the solid state and thus greatly diminish the tendency of adducts to form crystal. Meanwhile, the used polymer is photoresponsive, and its thin film can be readily cross-linked by UV irradiation to furnish fluorescent negative photoresist patterns with good resolution [24]. In this work, the ordered honeycomb structures are prepared by BF process, from TPE-containing polymer 1,2-dichloroethane solution, which have the AIE phenomenon.

Figure 2(a) is the fluorescence microscope image of the film before and after photopolymerization. From this image, we can see that the porous film can be prepared over a large area, and uniform pores with hexagonal arrangement are formed with a diameter of about $2-3 \mu \mathrm{m}$ and wall thickness of about $1.0 \mu \mathrm{m}$. The pore structure and the pore size are not affected by the light illumination. The porous film emits bright blue light before photopolymerization, while it emits dim blue light after photopolymerization. It illuminates that the photopolymerization makes the film emission intensity decrease. Figure 2(b) is fluorescent spectrum of the film before and after photopolymerization. When excited at $320 \mathrm{~nm}$, the honeycomb structure film emits strong blue light 


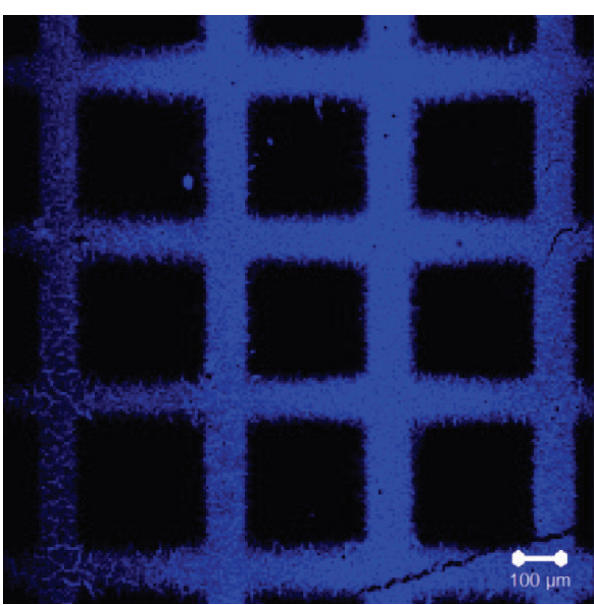

(a)

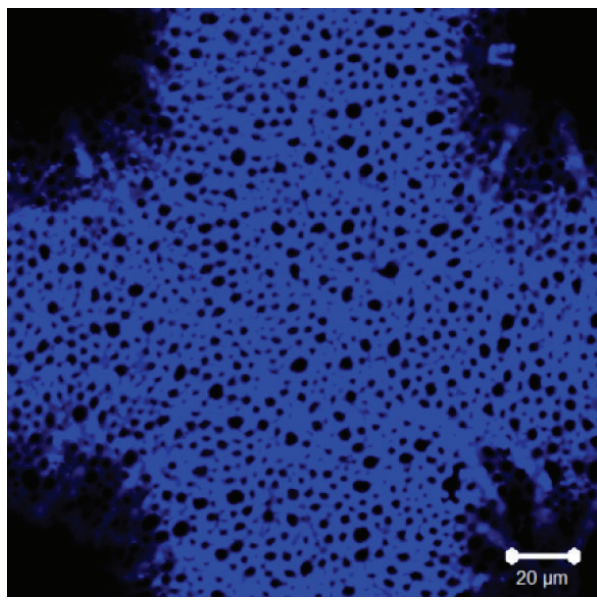

(c)

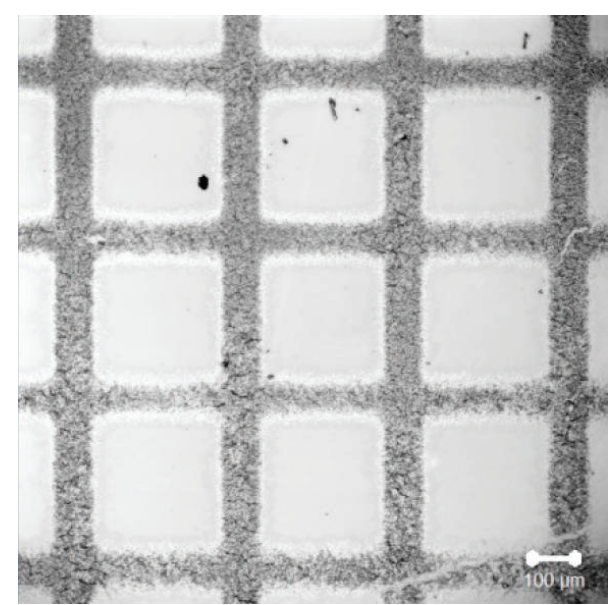

(b)

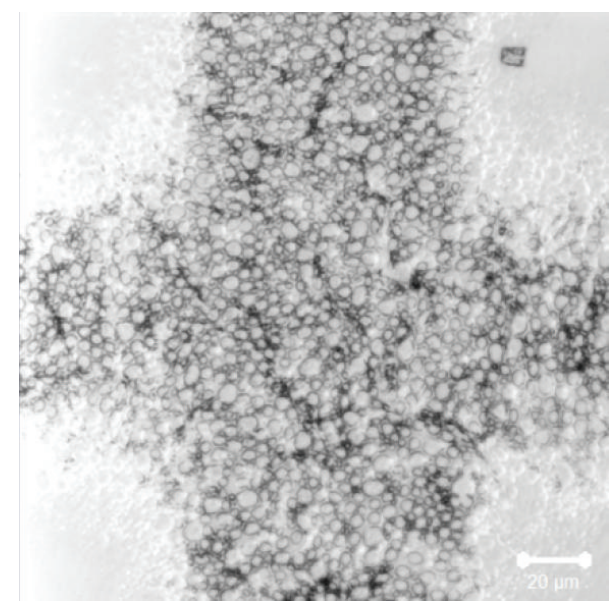

(d)

FIGURE 4: Confocal images of the patterned honeycomb square lattices: (a) low magnification, (c) high magnification, and the corresponding optical photos: (b) low magnification, (d) high magnification. In this figure, the size of the used mask is $100 \mu \mathrm{m}$ and the space is $300 \mu \mathrm{m}$.

with emission maxima at $482 \mathrm{~nm}$ before photopolymerization, while it emits weak blue light with emission maxima at $490 \mathrm{~nm}$ after photopolymerization, which is consistent with that observed in Figure 2(a). Thus, the photopolymerization leads to a red-shift of the photoluminescence of about $8 \mathrm{~nm}$. This is probably because the photopolymerization enhances intermolecular interaction, which leads to the red shift of the maximum fluorescence peak [25]. From the fluorescence microscope image and the fluorescent spectrum of the film before and after photopolymerization, it can be seen that the porous film has the AIE property whether it is photopolymerizable or not.

Figure 3(a) is low magnification confocal image of the patterned honeycomb square lattices. Figure 3(b) is the corresponding optical photo of the square lattices. From these images, it can be seen that the patterned square lattice film was successfully prepared. The blue light emission region with the size of $50 \mu \mathrm{m}$ is the honeycomb structure region, while the black region with the size of $150 \mu \mathrm{m}$ is the glass substrate region. The square lattice size in the confocal image seems to be larger than that in the optical photo because of the emissive effect. Figure 3(c) is the high magnification confocal image and Figure $3(\mathrm{~d})$ is the corresponding optical photo. From these images, it can be seen that the patterned square lattice film keeps the honeycomb structure very well. The diameter of the pore is about $2-3 \mu \mathrm{m}$ and the wall thickness is about $1.0 \mu \mathrm{m}$. The pore structure and the pore size are not affected by the photo-cross-linking process, which is consistent with that observed in Figure 2(a). Meanwhile, we also changed the used mask to prepare different size patterns, shown in Figure 4. Figure 4(a) is the low magnification confocal image of the patterned honeycomb square lattices, and Figure 4(b) is the corresponding optical photo. It can be seen that the patterned square lattices film was also successfully prepared. The blue light emission region with the size of $100 \mu \mathrm{m}$ is the honeycomb structure region, while the black region with the size of $300 \mu \mathrm{m}$ is the glass substrate region. Figure 4(c) is the high magnification confocal image, and Figure $4(\mathrm{~d})$ is the corresponding optical photo. From these images, it can be seen that the patterned square lattices film also keeps the honeycomb structure very well. The diameter of the pore is about $2-3 \mu \mathrm{m}$, and the wall thickness is about $1.0 \mu \mathrm{m}$. The pore 


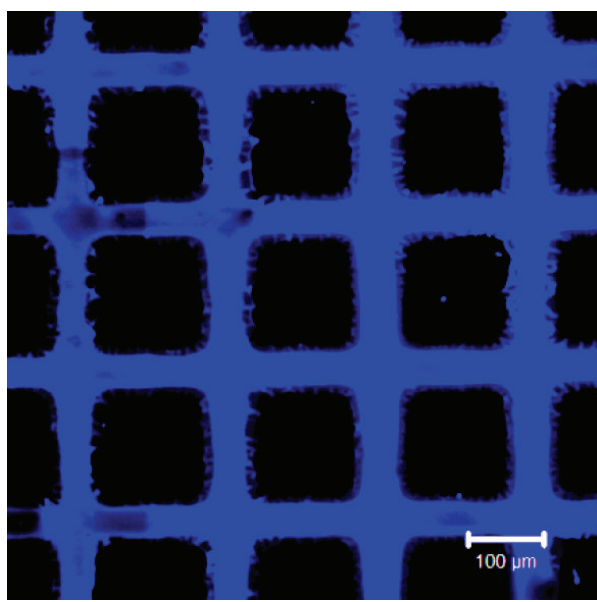

(a)

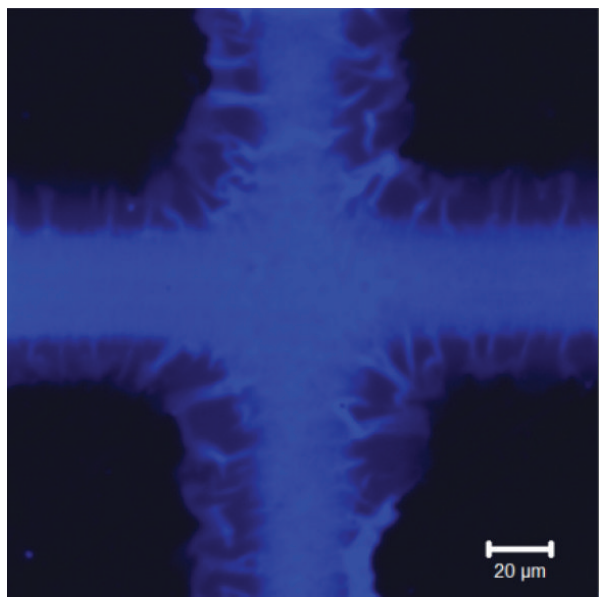

(c)

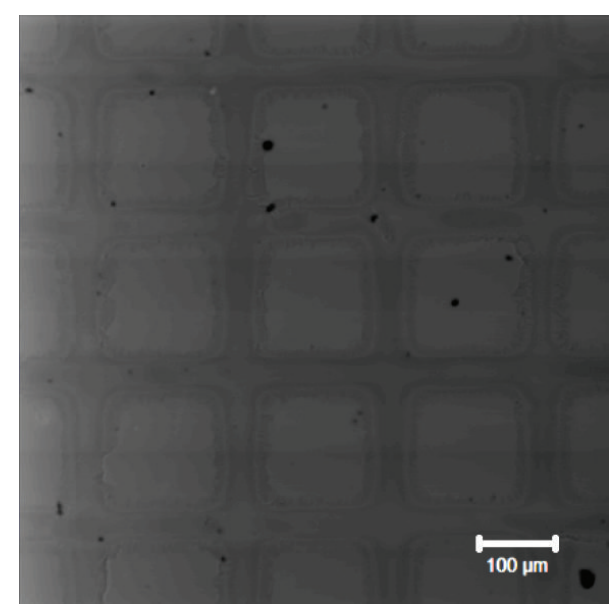

(b)

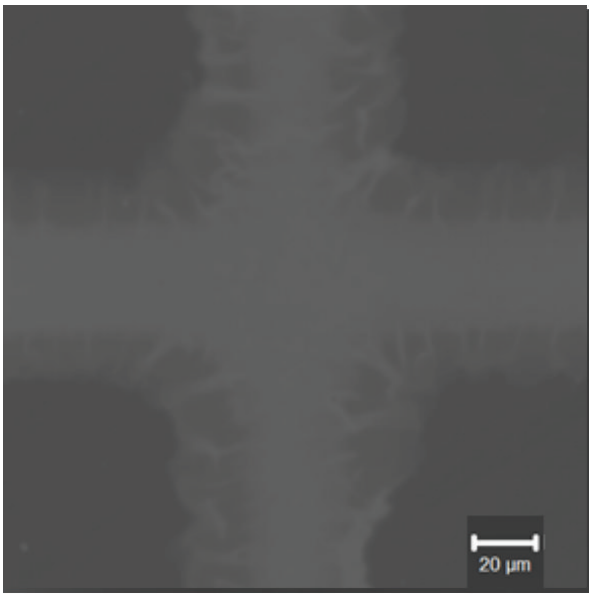

(d)

FIGURE 5: Confocal images of the patterned control smooth sample square lattices: (a) low magnification, (c) High magnification, and the corresponding optical photos: (b) low magnification, (d) high magnification. The size of the used mask is $50 \mu \mathrm{m}$ and the space is $150 \mu \mathrm{m}$.

structure and the pore size are also not affected by the photocross-linking process.

In control, we also prepared patterned smooth film. Figure 5(a) is the low magnification confocal image of patterned smooth film square lattices. Figure 5(b) is the corresponding optical photo of the square lattices. From these images, it can be seen that the patterned smooth film was successfully prepared, which has the same size of $50 \mathrm{um}$ pattern and the $150 \mathrm{um}$ space. The blue light emission region with the size of $50 \mu \mathrm{m}$ is the smooth film region, while the black region with the size of $150 \mu \mathrm{m}$ is the glass substrate region. Figure 5 (c) is the high magnification confocal image, and Figure $5(\mathrm{~d})$ is the corresponding optical photo. From these images, it can be seen that patterned square lattices film surface has no structures. Meanwhile, we also prepared different size patterns, shown in Figure 6. Figure 6(a) is the low magnification confocal image of the patterned smooth film square lattices, and Figure 6(b) is the corresponding optical photo. It can be seen that the patterned smooth film square lattices were also successfully prepared. The blue light emission region with the size of $100 \mu \mathrm{m}$ is the smooth film region, while the black region with the size of $300 \mu \mathrm{m}$ is the glass substrate region. Figure 6(c) is the high magnification confocal image, and Figure 6(d) is the corresponding optical photo. From these images, it can be seen that the patterned smooth film square lattices also have no structure on the surface.

In order to deeply understand how the honeycomb structure can affect the film wettability, the contact angles (CAs) of patterned honeycomb structure film, patterned smooth film (shown in Figure 7), honeycomb structure film, and smooth film (shown in Figure S1; see Supplementary Material available online at http://dx.doi.org/10.1155/2013/853154) were measured. The honeycomb structure films have the contact angle of $87.3 \pm 4.7^{\circ}$, and the smooth films have the contact angle of $28.6 \pm 3.2^{\circ}$, while the patterned honeycomb structure film with the size of $50 \mathrm{um}$ pattern and the $150 \mathrm{um}$ space has the CA of $91.5 \pm 2.8^{\circ}$, and the patterned honeycomb structure film with the size of $100 \mathrm{um}$ pattern and the $300 \mathrm{um}$ space has the CA of $92.3 \pm 3.2^{\circ}$. The patterned smooth film with the size of $50 \mathrm{um}$ pattern and the $150 \mathrm{um}$ space has the CA of $15.6 \pm 3.4^{\circ}$, and the patterned smooth film with the size 


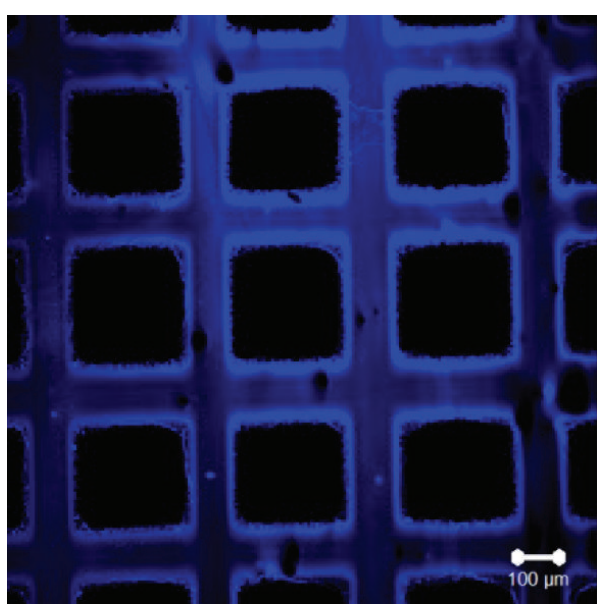

(a)

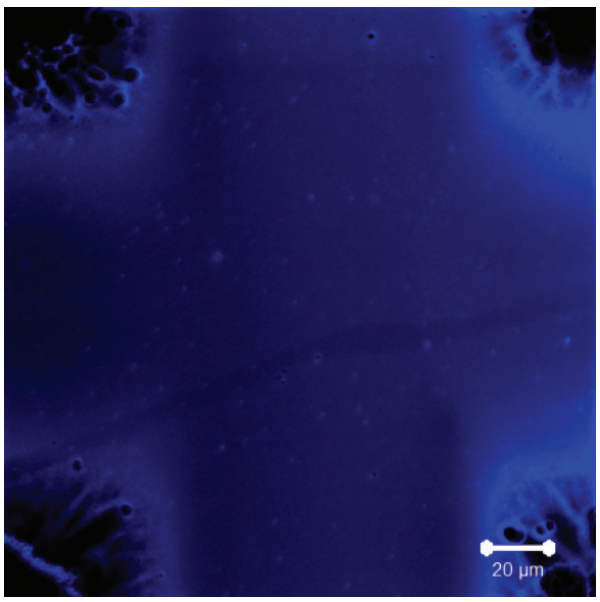

(c)

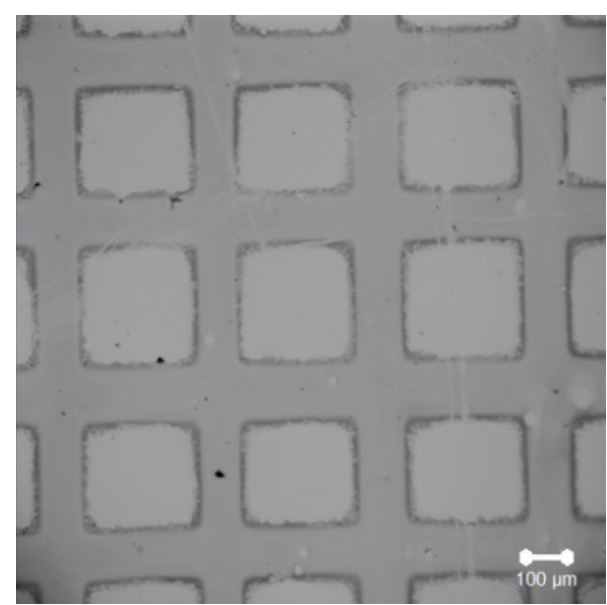

(b)

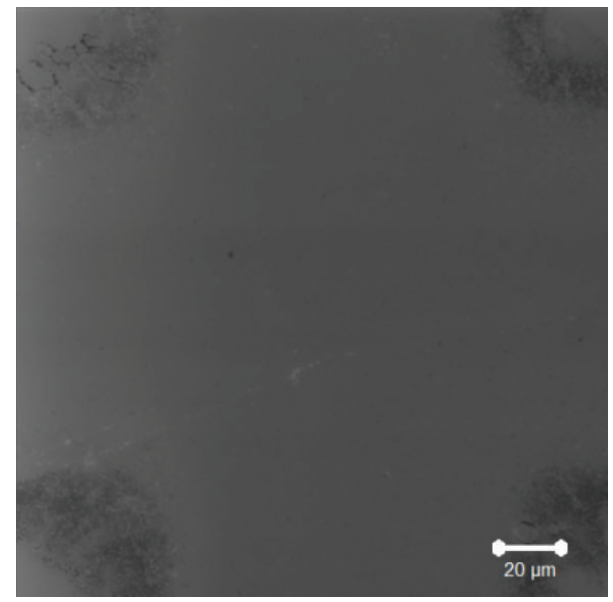

(d)

FIGURE 6: Confocal images of the patterned control smooth sample square lattices: (a) low magnification, (c) high magnification, and the corresponding optical photos: (b) low magnification, (d) high magnification. The size of the used mask is $100 \mu \mathrm{m}$ and the space is $300 \mu \mathrm{m}$.

of $100 \mathrm{um}$ pattern and the $300 \mathrm{um}$ space has the CA of $17.2 \pm$ $2.1^{\circ}$. In contrast with the honeycomb film and the smooth film, the contact angle of the patterned honeycomb structure film increased a little. While the patterned smooth film has a smaller one, this phenomenon illustrates that the CA will be decreased with the increases of the surface roughness by inducing patterns because the smooth film is hydrophilic.

To make the readers understand easily, the principle of the wetting on the solid surfaces was given. In 1936, Wenzel proposed a model to express the relationship between surface roughness and contact angle by the following equation:

$$
\operatorname{Cos} \theta_{W}=r \operatorname{Cos} \theta_{Y}
$$

where $r$ is the surface roughness factor, $\theta_{W}$ is the apparent contact angle in the Wenzel model, and $\theta_{Y}$ is the contact angle in Young's model [26]. The basic assumption in Wenzel's theory is that the liquids completely fill the grooves of the rough surface where they contact. From (1), it can be found that roughness enhances both wetting and antiwetting depending on the nature of the corresponding flat surface. If the smooth material gives a contact angle greater than $90^{\circ}$, the presence of surface roughness increases this angle still further, but if $\theta$ is less than $90^{\circ}$, the surface roughness decreases the angle.

As cast, smooth film shows hydrophilicity (CA $=28 \pm$ $3.2^{\circ}$ ). Wenzel's theory indicates that the increase of roughness of a hydrophilic solid surface will result in more hydrophilic surface [26]. From the fluorescence microscope image (Figure 2(a)), it can be seen that the pores on the film are formed and separated from each other. Therefore, an air pocket at the interface between water and honeycomb structure film can be formed. The presence of air pores in the honeycomb film can improve the roughness of the surface. If the honeycomb film is of the same chemical composition with that of the smooth surface, the surface topography will be one of dominant factors for the control wettability. The CA of the honeycomb film will become small according to Wenzel's theory. Actually, the CA data increased at the honeycomb film surface. So, there must be another factor to play an important role, which is the change of the surface chemical composition. The CA change is mainly caused by the change of the surface roughness 


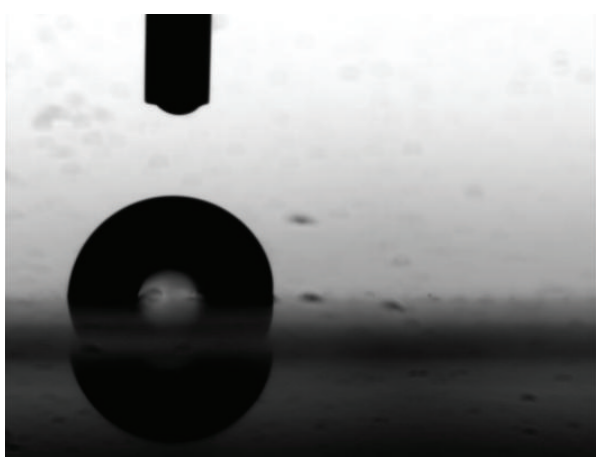

(a)

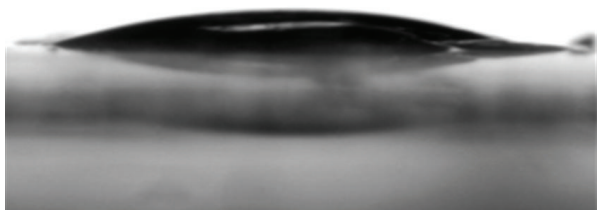

(c)

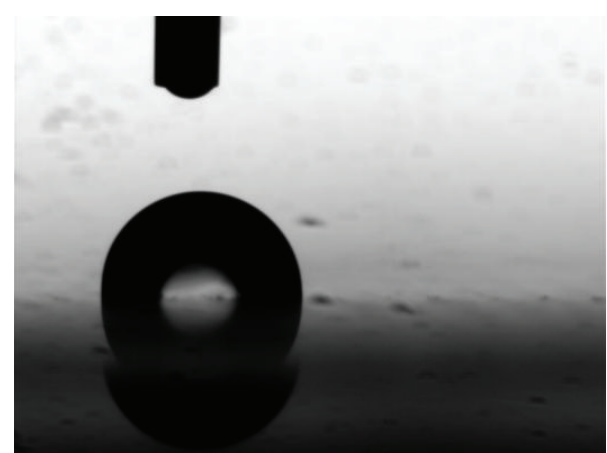

(b)

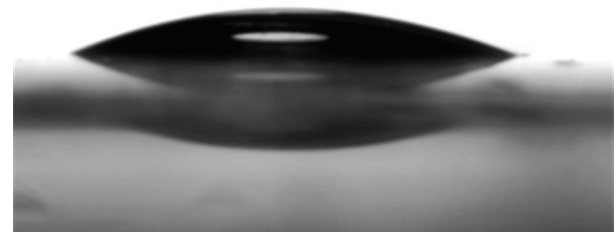

(d)

FIGURE 7: Photographs of water droplet shape. (a) Patterned honeycomb structure film with the size of 50 um pattern and the 150 um space has the CA of about $91.5 \pm 2.8^{\circ}$; (b) the patterned honeycomb structure film with the size of 100 um pattern and the 300 um space has the CA of about $92.3 \pm 3.2^{\circ}$; (c) the patterned smooth film with the size of 50 um pattern and the 150 um space has the CA of about $15.6 \pm 3.4^{\circ}$; and (d) the patterned smooth film with the size of 100 um pattern and the 300 um space has the CA of about $17.2 \pm 2.1^{\circ}$.

and the surface free energy [26, 27]. During the BF process, the hydrophilic blocks tend to aggregate themselves around the water droplets because of the mutual interaction. After the evaporation of water, porous films with polar functional groups enriched inside the pores and hydrophobic blocks enriched on the external surface were achieved [28-30]. The formation process of the honeycomb film by BF method makes the hydrophilic group of the polymer concentrate in the pore wall, and the hydrophobic group aggregate on the film surface [31]. The surface free energy decreases because of the chemical composition change. So, the increase of the roughness and the decrease of the surface free energy are responsible for the surface wettability change. Furthermore, the honeycomb film is hydrophobic with a CA of about 90, so the CA will be increased with the increases of the surface roughness by inducing patterns.

\section{Conclusion}

The patterned porous honeycomb-like film with the AIE property was prepared successfully by the BF method. Characterization of contact angles (CA) indicates that the patterned porous honeycomb structure can improve the hydrophobicity of the film, while smooth films do not have this function. Characterization of the fluorescence property indicates that the patterned honeycomb structure film is highly emissive. This work could not only be promising for controlling molecular group reorientation and suitable for the application of manipulating surface composition of the film but also make the TPE derivatives with AIE property have a promising application future in special wettability surface. The present findings should open a way for the new application of honeycomb structure materials, which will be useful in many areas, such as sensors, tissue engineering, clinical medicine, and biomaterials.

\section{Acknowledgments}

This work was supported by the National Research Fund for Fundamental Key Projects (2013CB834705, 2010CB934700, and 2011CB935700) and the National Natural Science Foundation of China (Grant no. 21003138).

\section{References}

[1] G. Widawski, M. Rawiso, and B. François, "Self-organized honeycomb morphology of star-polymer polystyrene films," Nature, vol. 369, no. 6479, pp. 387-389, 1994.

[2] N. Maruyama, O. Karthaus, K. Ijiro et al., "Mesoscopic pattern formation of nanostructured polymer assemblies," Supramolecular Science, vol. 5, no. 3-4, pp. 331-336, 1998.

[3] B. de Boer, U. Stalmach, P. F. van Hutten, C. Melzer, V. V. Krasnikov, and G. Hadziioannou, "Supramolecular selfassembly and opto-electronic properties of semiconducting block copolymers," Polymer, vol. 42, no. 21, pp. 9097-9109, 2001. 
[4] L. V. Govor, I. A. Bashmakov, R. Kiebooms, V. Dyakonov, and J. Parisi, "Self-organized networks based on conjugated polymers," Advanced Materials, vol. 13, no. 8, pp. 588-591, 2001.

[5] T. Nishikawa, R. Ookura, J. Nishida et al., "Fabrication of honeycomb film of an amphiphilic copolymer at the air-water interface," Langmuir, vol. 18, no. 15, pp. 5734-5740, 2002.

[6] T. Nishikawa, J. Nishida, R. Ookura et al., "Web-structured films of an amphiphilic polymer from water in oil emulsion: fabrication and characterization," Langmuir, vol. 16, no. 3, pp. 1337-1342, 2000.

[7] J. H. Kim, M. Seo, and S. Y. Kim, "Lithographically patterned breath figure of photoresponsive small molecules: dualpatterned honeycomb lines from a combination of bottom-up and top-down lithography," Advanced Materials, vol. 21, no. 41, pp. 4130-4133, 2009.

[8] L. A. Connal, R. Vestberg, C. J. Hawker, and G. G. Qiao, "Dramatic morphology control in the fabrication of porous polymer films," Advanced Functional Materials, vol. 18, no. 22, pp. 3706-3714, 2008.

[9] L. P. Heng, J. Zhai, Y. Zhao, J. J. Xu, X. L. Sheng, and L. Jiang, "Enhancement of photocurrent generation by honeycomb structures in organic thin films," ChemPhysChem, vol. 7, no. 12, pp. 2520-2525, 2006.

[10] K. Kon, C. N. Brauer, K. Hidaka, H. Löhmannsröben, and O. Karthaus, "Preparation of patterned zinc oxide films by breath figure templating," Langmuir, vol. 26, no. 14, pp. 12173-12176, 2010.

[11] M. S. Park and J. K. Kim, "Broad-band antireflection coating at near-infrared wavelengths by a breath figure," Langmuir, vol. 21, no. 24, pp. 11404-11408, 2005.

[12] H. Yabu, M. Takebayashi, M. Tanaka, and M. Shimomura, "Superhydrophobic and lipophobic properties of self-organized honeycomb and pincushion structures," Langmuir, vol. 21, no. 8, pp. 3235-3237, 2005.

[13] X. Xu, L. P. Heng, X. J. Zhao, J. Ma, L. Lin, and L. Jiang, "Multiscale bio-inspired honeycomb structure material with high mechanical strength and low density," Journal of Materials Chemistry, vol. 22, pp. 10883-10888, 2012.

[14] L. P. Heng, B. Wang, M. C. Li, Y. Q. Zhang, and L. Jiang, "Advances in fabrication materials of honeycomb structure films by the breath-figure method," Materials, vol. 6, no. 2, pp. 460-482, 2013.

[15] T. Nishikawa, J. Nishida, R. Ookura et al., "Mesoscopic patterning of cell adhesive substrates as novel biofunctional interfaces," Materials Science and Engineering C, vol. 10, no. 1-2, pp. 141-146, 1999.

[16] M. Tanaka, "Design of novel 2D and 3D biointerfaces using selforganization to control cell behavior," Biochimica et Biophysica Acta, vol. 1810, no. 3, pp. 251-258, 2011.

[17] T. Nishikawa, M. Nonomura, K. Arai et al., "Micropatterns based on deformation of a viscoelastic honeycomb mesh," Langmuir, vol. 19, no. 15, pp. 6193-6201, 2003.

[18] R. H. Friend, R. W. Gymer, A. B. Holmes et al., "Electroluminescence in conjugated polymers," Nature, vol. 397, no. 6715, pp. 121-128, 1999.

[19] Z. Zhao, Z. Wang, P. Lu et al., "Structural modulation of solidstate emission of 2,5-bis(trialkylsilylethynyl)-3,4-diphenylsiloles," Angewandte Chemie-International Edition, vol. 48, no. 41, pp. 7608-7611, 2009.

[20] W. Z. Yuan, P. Lu, S. Chen et al., "Changing the behavior of chromophores from aggregation-caused quenching to aggregationinduced emission: development of highly efficient light emitters in the solid state," Advanced Materials, vol. 22, no. 19, pp. 21592163, 2010.

[21] J. Z. Liu, J. W. Y. Lam, and B. Z. Tang, "Acetylenic polymers: syntheses, structures, and functions," Chemical Reviews, vol. 109, no. 11, pp. 5799-5867, 2009.

[22] Y. N. Hong, J. W. Y. Lam, and B. Z. Tang, "Aggregationinduced emission: phenomenon, mechanism and applications," Chemical Communications, no. 29, pp. 4332-4353, 2009.

[23] J. Z. Liu, J. W. Y. Lam, and B. Z. Tang, "Aggregation-induced emission of silole molecules and polymers: fundamental and applications," Journal of Inorganic and Organometallic Polymers and Materials, vol. 19, no. 3, pp. 249-285, 2009.

[24] R. R. Hu, J. W. Y. Lam, Y. Yu et al., "Facile synthesis of soluble nonlinear polymers with glycogen-like structures and functional properties from "simple" acrylic monomers," Polymer Chemistry, vol. 4, no. 1, pp. 95-105, 2013.

[25] L. P. Heng, W. Qin, S. J. Chen et al., "Fabrication of small organic luminogens honeycomb-structured films with aggregationinduced emission features," Journal of Materials Chemistry, vol. 22, pp. 15869-15873, 2012.

[26] R. N. Wenzel, "Resistance of solid surfaces to wetting by water," Industrial and Engineering Chemistry, vol. 28, no. 8, pp. 988994, 1936.

[27] A. B. D. Cassie and S. Baxter, "Wettability of porous surfaces," Transactions of the Faraday Society, vol. 40, pp. 546-551, 1944.

[28] B. B. Ke, L. S. Wan, and Z. K. Xu, "Controllable construction of carbohydrate microarrays by site-directed grafting on selforganized porous films," Langmuir, vol. 26, no. 11, pp. 89468952, 2010.

[29] F. Galeotti, V. Calabrese, M. Cavazzini et al., "Self-functionalizing polymer film surfaces assisted by specific polystyrene endtagging," Chemistry of Materials, vol. 22, no. 9, pp. 2764-2769, 2010.

[30] M. H. Stenzel and T. P. Davis, "Biomimetic honeycombstructured surfaces formed from block copolymers incorporating acryloyl phosphorylcholine," Australian Journal of Chemistry, vol. 56, no. 10, pp. 1035-1038, 2003.

[31] P. C. Chen, L. S. Wan, B. B. Ke, and Z. K. Xu, "Honeycombpatterned film segregated with phenylboronic acid for glucose sensing," Langmuir, vol. 27, no. 20, pp. 12597-12605, 2011. 

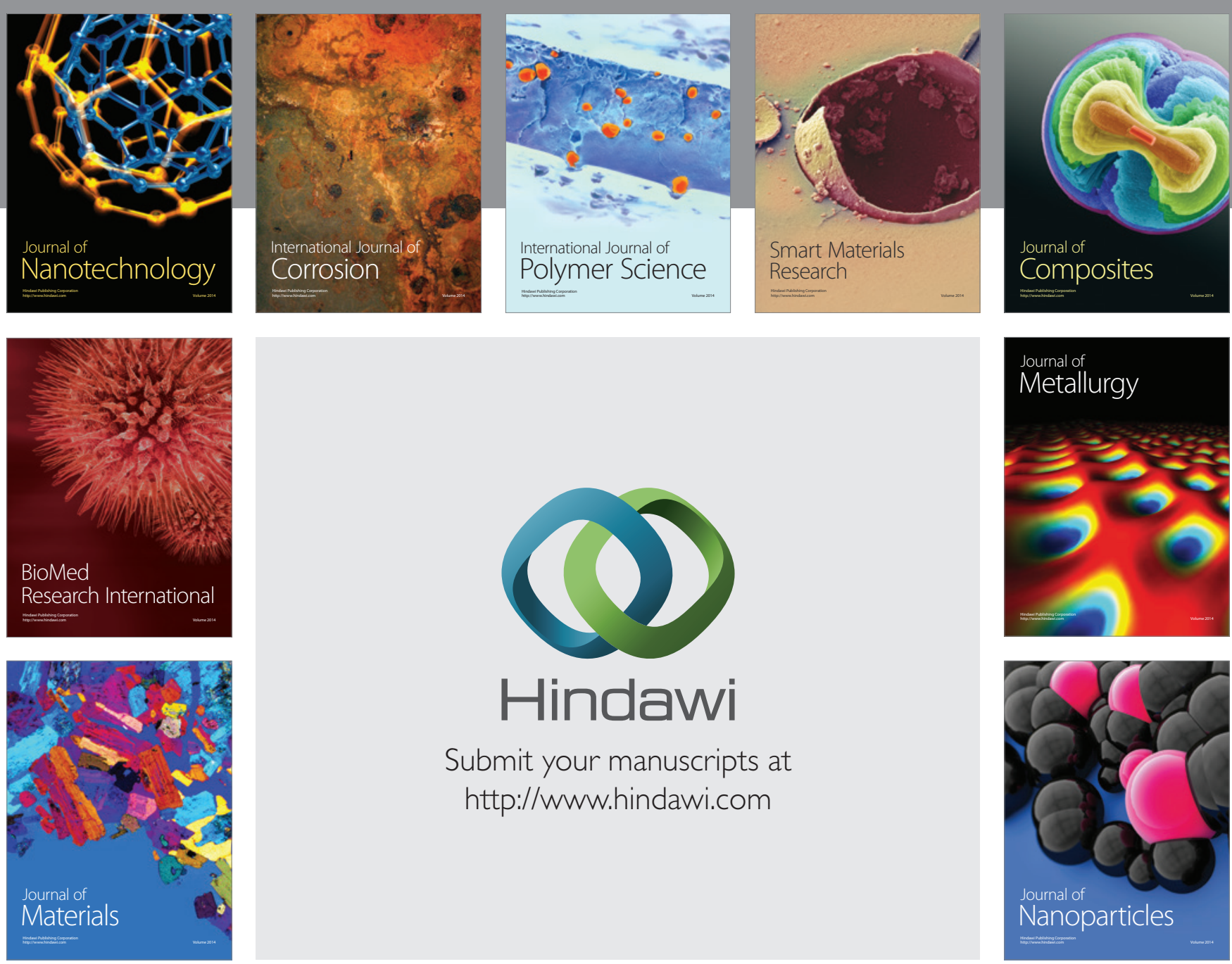

Submit your manuscripts at http://www.hindawi.com
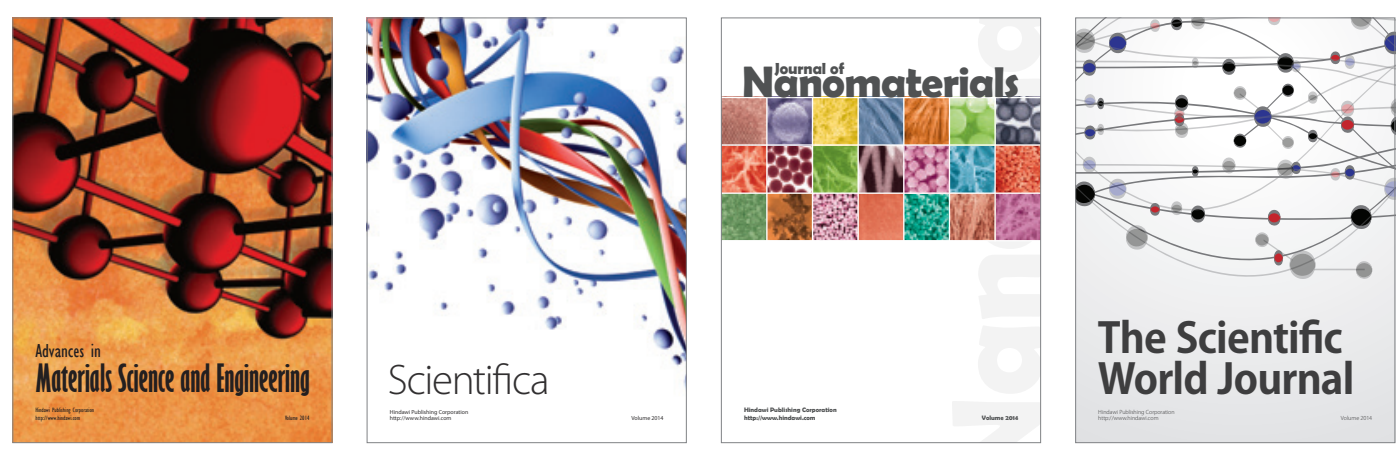

\section{The Scientific World Journal}
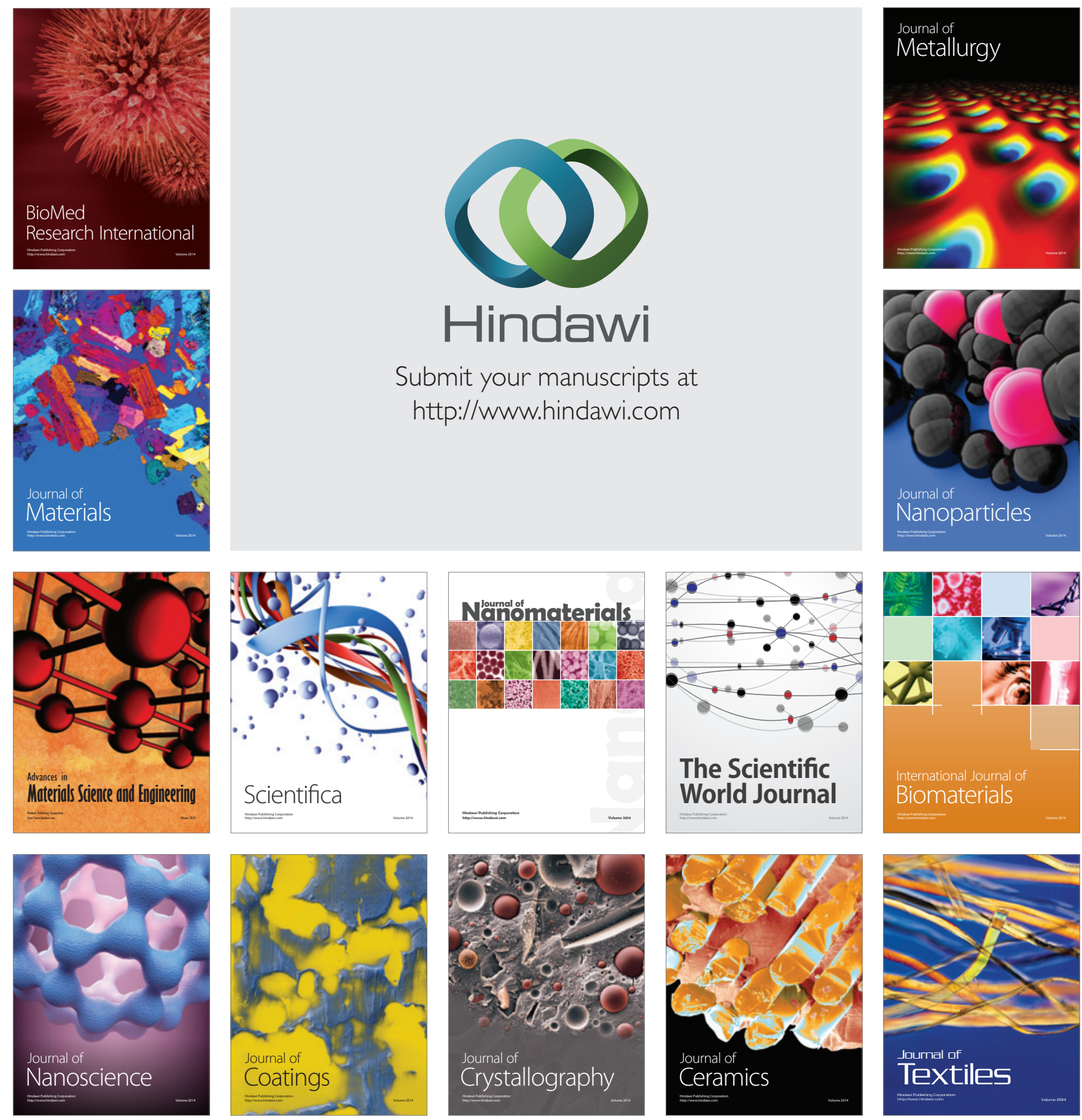